DOI: 10.12957/demetra.2016.20064

\title{
Estado nutricional, sinais clínicos de carências nutricionais e vulnerabilidade social entre crianças do semiárido paraibano
}

\section{Nutritional status, clinical signals of nutritional deficiencies and social vulnerability among children from the semi-arid region of Paraiba}

\author{
José Lucas Braga Santos' \\ Poliana de Araújo Palmeira' \\ Vanille Valério Barbosa Pessoa Cardoso \\ Marília Ferreira Frazão $0^{\top}$ \\ 1 Universidade Federal de Campina Grande, \\ Núcleo de Pesquisa e Estudos em Nutrição e \\ Saúde Coletiva - Núcleo PENSO. Sítio Olho \\ d'agua da bica, Cuite-PB, Brasil. \\ Correspondência/Correspondence \\ José Lucas Braga Santos \\ E-mail: joselucasbragasantos@hotmail.com
}

\section{Resumo}

Objetivo: analisar o estado nutricional antropométrico e sinais clínicos de carências nutricionais segundo condições socioeconômicas de crianças com idade entre 0 e 9 anos matriculadas em creches e escolas públicas municipais de Cuité, Paraíba. Metodologia: Estudo transversal com amostra por conveniência composta por 629 crianças. Coletou-se informações sociais da família, classificadas segundo linha da pobreza. Foram aferidas medidas antropométricas e realizada semiologia nutricional, para determinação do estado nutricional. Resultados: $\mathrm{O}$ excesso de peso prevaleceu sobre o baixo peso em ambos os grupos de renda. Verificou-se a presença de 3 a 5 sinais de alterações clínicas relacionadas às carências nutricionais em cerca de $20 \%$ das crianças que conviviam com famílias acima e abaixo da linha da pobreza. Entretanto, entre as crianças que apresentaram de 3 a 5 sinais carenciais e estavam inclusas em famílias abaixo da linha da pobreza, observou-se maior percentual de déficit de peso $(19,5 \%)$ do que de excesso $(15,6 \%)$. Para as crianças de famílias acima da linha da pobreza com as mesmas alterações clínicas, a prevalência de excesso de peso $(20 \%)$ revelou-se expressivamente maior que a de baixo peso $(8,9 \%)$, mostrando a presença de sinais de carências nutricionais em crianças com características de peso opostas. Conclusão: Assim, para condições distintas de vulnerabilidade social pôde-se perceber a presença de uma dupla carga de doenças com origem na alimentação: o excesso de peso e carência de nutrientes específicos, o que é típico do processo de transição nutricional.

Palavras-chave: Estado nutricional. Deficiências Nutricionais. Crianças. Pobreza. 


\section{Abstract}

Objective: To analyze the anthropometric nutritional status and clinical signs of nutritional deficiencies by social vulnerability status of children aged 0-9 years enrolled in day care centers and municipal public schools in the city of Cuité, Paraíba. Methods: A cross-sectional study with the convenience sample composed by 629 children. Family social information was collected and classified according to the poverty line. Anthropometric measurements were performed and nutritional semiology was performed to determine nutritional status. Results: The prevalence of overweight children prevailed underweight children between both of income groups analyzed. It was possible to verify the presence of 3 to 5 signals of clinical alterations related with nutritional deficiencies in about $20 \%$ of children who live in families above and below the poverty line. However, It was verified that children who show from 3 to 5 deficiency signs and are included in families who live below the poverty line presented higher weight deficit percentage (19,5\%) than overweight $(15,6 \%)$. For children from families who live above the poverty line with the same clinical alterations, the prevalence of overweight (20\%) was significantly higher than underweight $(8,9 \%)$, proving that the presence of signs of nutritional deficiencies are also present in children with opposite weight characteristics. Conclusion: Therefore, for different conditions of social vulnerability, it is possible to perceive the presence of a double burden of diseases derived from eating habits: The overweight and the deficiency of specific nutrients, typical characteristics from the contemporary process of the Brazilian nutritional transition.

Key words: Nutritional Status. Nutritional deficiencies. Child. Poverty.

\section{Introdução}

A Pesquisa de Orçamentos Familiares (POF) 2008-2009 realizada pelo Instituto Brasileiro de Geografia e Estatística, em parceria com o Ministério da Saúde, apresentou como resultado um aumento importante no número de crianças acima do peso no Brasil, principalmente na faixa etária entre 5 e 9 anos de idade. A prevalência de meninos acima do peso mais que triplicou entre 1989 e 2009, passando de 8,7\% para 30,3\%, respectivamente. Entre as meninas esta variação foi de 7,5\% para 26,5\% em 2009. Quanto ao déficit de peso, as prevalências não ultrapassaram, em média, 2,5\% em ambos os sexos entre 1989 e 2009. No tocante ao déficit de altura na faixa etária de 5 a 9 anos a prevalência foi de $6,8 \%$, tendendo a diminuir com a idade. ${ }^{1}$ 
Apesar da diminuição da desnutrição, $\operatorname{estudos}^{2,3}$ têm mostrado que a redução da prevalência das carências nutricionais não acontece com a mesma velocidade, ou seja, atrelado ao aumento de sobrepeso e obesidade na população brasileira verifica-se também grande privação do consumo de micronutrientes, que caracteriza o desenvolvimento de deficiências nutricionais específicas, que por muito tempo estiveram relacionadas com a desnutrição em crianças. Trata-se de duas situações que se agravam simultaneamente, mas que são opostas por definição: carência nutricional e obesidade, condição típica dos excessos alimentares. ${ }^{4}$

Estudos recentes sobre deficiências de micronutrientes, especialmente em crianças, são escassos. Destacam-se os achados de Fidelis \& Osório ${ }^{5}$ em um estudo com crianças de até 5 anos de idade a partir de recordatório alimentar de 24 horas, em que se observou que a prevalência de inadequação dos micronutrientes foi elevada, principalmente de ferro e zinco, naquelas acima de 12 meses de idade. Outro estudo, realizado por Pedraza et al. ${ }^{6}$, em 2013, no Estado da Paraíba, com crianças matriculadas em creches públicas, a partir da observação dos níveis séricos de micronutrientes, constatou que 23,3\% das crianças apresentavam deficiência de vitamina A, 15,4\% eram anêmicas e 13,8\% manifestaram deficiência de zinco. Nesse sentido, cabe ressaltar que condições socioeconômicas e ambientais como renda, saneamento, habitação, acesso à água e outros, são responsáveis por uma parcela significativa da situação de saúde infantil. ${ }^{7}$

O presente estudo objetivou analisar o estado nutricional antropométrico e os sinais clínicos de deficiências nutricionais segundo condições socioeconômicas de crianças com idade entre 0 e 9 anos matriculadas em creches e escolas públicas municipais da zona urbana de Cuité, Paraíba.

Espera-se, portanto, subsidiar com o conhecimento sobre a distribuição e magnitude de problemas nutricionais em crianças e assim incentivar a proposição de ações práticas de saúde no campo do planejamento e da elaboração de intervenções educativas e de assistência sobre o controle e prevenção de problemas nutricionais.

\section{Metodologia}

\section{População e coleta de dados}

Este é um estudo transversal com amostragem por conveniência, que compreendeu a realização de uma Chamada Nutricional com crianças regularmente matriculadas em creches e escolas municipais da zona urbana do município de Cuité, Paraíba. A Chamada Nutricional é uma estratégia de estimativa rápida populacional frequentemente aplicada em populações específicas em condição de vulnerabilidade e para fins de vigilância, executada num período curto de tempo e com custo reduzido. ${ }^{8}$ 
Os pesquisadores foram alunos de graduação em nutrição da Universidade Federal de Campina Grande, selecionados a partir do interesse e disponibilidade em participar da pesquisa, e previamente treinados, com carga horária de 18 horas.

A estratégia de trabalho de campo foi concebida com o apoio dos gestores e diretores das escolas e a coleta de dados, realizada no âmbito escolar. Foi acordado anteriormente as datas e horários com cada uma das escolas, onde foram organizados os equipamentos necessários para a aferição de medidas antropométricas.

A coleta de dados foi feita entre os meses de maio e junho de 2013. Com base no censo escolar de 2012, registrou-se 2.888 alunos regularmente matriculados na rede municipal de ensino de Cuité, sendo 1.843 em escolas e creches da zona urbana. Com base nesse levantamento, foi planejado o deslocamento da equipe de pesquisa até as escolas, e os responsáveis pelos alunos foram orientados a comparecer ao estabelecimento para contribuir com o preenchimento de informações sobre condições de vida da família.

Ao final do trabalho de campo, do total de alunos matriculados, 1.562 foram pesquisados. Para este estudo, considerou-se avaliar apenas os estudantes classificados como crianças, ${ }^{11}$ que compreende a faixa etária de 0 a 9 e 11 meses de idade, totalizando 782 crianças. A amostra final deste estudo é composta por 629 crianças, cujos responsáveis compareceram à escola no dia da pesquisa e autorizaram a participação por meio da assinatura do Termo de Consentimento Livre e Esclarecido, bem como responderam ao questionário socioeconômico.

\section{Características ambientais e socioeconômicas}

Foi aplicado um questionário contendo informações sobre o domicílio e o responsável da família: Área de moraria (urbana e rural), Esgotamento sanitário (Rede pública/fossa séptica, Fossa rudimentar/vala ou Esgoto a céu aberto), Disponibilidade diária de água no domicílio (Sim ou não), Ocupação (ter ou não ter trabalho) e Escolaridade do responsável (baixa escolaridade, quando o indivíduo relatou ter cursado até o ensino médio incompleto, e alta escolaridade quando declarou ter cursado no mínimo o ensino médio completo). Para a composição da variável renda da família, foram coletados os valores de renda para cada membro da família e o número de moradores do domicílio.

\section{Antropometria e semiologia nutricional}

A aferição do peso foi realizada com balança digital de vidro ultraSLIM - w903 - WISO. A criança foi pesada descalça, com a menor quantidade de roupa possível e sem a presença de 
objetos no bolso, mãos na cabeça e no centro da plataforma da balança, com os braços ao longo do corpo. A aferição da altura dos menores de um ano de idade foi feita por um estadiômetro infantil de madeira com a criança deitada em local apropriado disponível nas creches. Para os demais foi utilizada uma fita métrica com precisão de $0,1 \mathrm{~cm}$ fixada na parede sem rodapé e com superfície lisa, com o escolar descalço e sem qualquer objeto na cabeça, posicionado de costas, com os calcanhares encostados à parede, como recomendado no Manual Técnico do Sistema de Vigilância Alimentar e Nutricional - SISVAN. ${ }^{9}$

A fim de detectar a presença de sinais da anemia e da deficiência de outros nutrientes específicos, como zinco, proteína, ácido fólico e vitamina A, foi realizado o exame físico de cada criança, considerando a coloração da pele da região palmar e das mucosas, principalmente a mucosa interna dos olhos. Além desses indicadores, observou-se na semiologia nutricional a cavidade oral das crianças quanto à presença de feridas e alterações tróficas na pele, pelos e fâneros, bem como a presença de edema nos membros inferiores e distensão abdominal. ${ }^{10}$

\section{Análise de dados}

Após a coleta dos dados, os questionários foram digitados utilizando os recursos do programa Microsoft Access em computadores instalados no Laboratório de Informática Aplicada do Centro de Educação e Saúde. Após a digitação, o banco de dados foi transferido para o Programa SPSS for Windows versão 13.0, para a realização da análise estatística descritiva dos dados, nos computadores da sala do Núcleo de Pesquisas e Estudos em Nutrição e Saúde Coletiva - PENSO.

A renda mensal familiar per capita foi adotada como variável independente, sendo a família da criança classificada com renda acima ou abaixo da linha da pobreza, de acordo com o ponto de corte de inferior ou superior a $\mathrm{R} \$ 140,00$ reais mensais per capita, valor estabelecido para a classificação da pobreza no Programa Bolsa Família. ${ }^{12}$

As variáveis dependentes se referem ao estado nutricional da criança. O estado nutricional foi avaliado a partir de dois indicadores mais largamente usados no campo científico para avaliação e discussão de um conjunto de crianças, $\mathrm{P} / \mathrm{I}$ e A/I, visto a maior sensibilidade à classificação do estado nutricional na faixa etária da amostra. ${ }^{13}$

A partir da análise das curvas de crescimento da World Health Organization, ${ }^{13}$ as crianças que, pelo indicador $\mathrm{P} / \mathrm{I}$, encontravam-se em situação de risco de sobrepeso, sobrepeso ou obesidade ( $\geq$ escore- $z+1$ e $>$ escore- $z+3$ ) foram classificadas em Excesso de peso, e aquelas em risco de baixo peso, baixo peso ou peso extremo para idade ( $\geq$ escore-z $-2 \quad \mathrm{e}<$ escore- $\mathrm{z}-3$ ), em Baixo peso. Da mesma forma para o indicador $\mathrm{A} / \mathrm{I}$, quando encontradas em situação de risco ou baixa estatura ( $\geq$ escore-z -2 e $<$ escore- $z-2$ ), as crianças foram classificadas em Baixa estatura, e quando em risco ou estatura elevada ( $>$ escore- $z+2$ e $\leq$ escore- $z+2$ ), em Estatura elevada. 
Com relação aos sinais clínicos de carências nutricionais, foram avaliadas nove variáveis: aspectos unhas (normal ou alteração), cabelos (normal, ressecado, quebradiço, ou com queda) e pele (ressecada, áspera, manchas ou erupções cutâneas); palidez palmar e da mucosa interna dos olhos; presença de edema em membros inferiores; distensão abdominal; ferida em canto de boca e Mancha de Bitot. Cada variável foi categorizada segundo a existência ou não de qualquer alteração clínica, e posteriormente, para cada criança, foi somada a quantidade de alterações clínicas observadas, podendo variar de 1 a 8.

Considerando a distribuição da frequência dessas alterações na própria amostra, observou-se, no máximo, cinco alterações clínicas por criança. Estas foram agrupadas seguindo um critério de menor ou maior característica física e clínica relacionada com carência nutricional, adotado pelos pesquisadores. Nesse sentido, avaliou-se que as crianças que apresentavam 3 a 5 alterações clínicas reuniriam um conjunto de condições clínicas maior para identificar carência nutricional.

Para todas as análises estatísticas dos dados, o teste de significância utilizado foi o Qui-quadrado, considerando o valor de $\mathrm{p}<0,05$.

\section{Aspectos éticos}

O projeto foi submetido e aprovado pelo Comitê de Ética em Pesquisa do Hospital Universitário Alcides Carneiro, da Universidade Federal de Campina Grande (UFCG), CAAE: 15713713.0.0000.5182. Para realizar a coleta de dados nas escolas, a secretária municipal de Educação assinou o Termo de Compromisso, concordando com o desenvolvimento da pesquisa.

O escolar e o responsável foram convidados pelos entrevistadores a participar da Chamada Nutricional, onde se explicou os objetivos da pesquisa, e em caso de aceite, o responsável assinou o Termo de Consentimento Livre e Esclarecido.

\section{Resultados}

Os escolares estudados residem em um município de pequeno porte localizado no semiárido nordestino. Segundo o Índice de Desenvolvimento Humano, o município Cuité é classificado como de baixo desenvolvimento (0.591), o que expressa a vulnerabilidade social, de saúde, trabalho e renda da população desse município. Com a análise dos dados, reforça-se esta vulnerabilidade, visto que $62,2 \%$ dos escolares estudados residiam entre famílias classificadas como abaixo da linha da pobreza.

A Tabela 1 apresenta as características sociais e demográficas das famílias e dos responsáveis pelas crianças estudadas. Quanto aos responsáveis pela criança, constatou-se um maior percentual 
com alta escolaridade entre as famílias classificadas acima da linha da pobreza. Observou-se ainda percentuais semelhantes de acesso ao esgotamento sanitário e à água, evidenciando assim que a vulnerabilidade ambiental alcançou tanto as famílias que estavam abaixo como aquelas acima da linha da pobreza.

Tabela 1. Características sociais e demográficas das famílias, segundo situação de vulnerabilidade social, Cuité-PB, 2012.

\begin{tabular}{|c|c|c|c|c|c|c|c|}
\hline \multirow[t]{2}{*}{ Variáveis } & \multicolumn{2}{|c|}{ Total } & \multicolumn{2}{|c|}{$\begin{array}{l}\text { Famílias } \\
\text { acima da } \\
\text { linha da } \\
\text { pobreza }\end{array}$} & \multicolumn{2}{|c|}{$\begin{array}{l}\text { Famílias } \\
\text { abaixo da } \\
\text { linha da } \\
\text { pobreza }\end{array}$} & \multirow[t]{2}{*}{$\begin{array}{l}\text { Valor } \\
\text { de } P\end{array}$} \\
\hline & $\mathrm{N}$ & $\%$ & $\mathrm{~N}$ & $\%$ & $\mathrm{~N}$ & $\%$ & \\
\hline \multicolumn{8}{|l|}{ Área } \\
\hline Urbano & 583 & 92,7 & 220 & 92,9 & 361 & 92,6 & \multirow{2}{*}{, 884} \\
\hline Rural & 46 & 7,3 & 17 & 7,1 & 29 & 7,4 & \\
\hline \multicolumn{8}{|l|}{ Esgotamento sanitário ${ }^{1}$} \\
\hline Rede pública/Fossa séptica & 196 & 31,8 & 64 & 27,5 & 132 & 34,4 & \multirow{3}{*}{,201 } \\
\hline Fossa rudimentar/Vala & 392 & 63,5 & 157 & 67,4 & 235 & 61,2 & \\
\hline Não tem/Esgoto a céu aberto & 29 & 4,7 & 12 & 5,2 & 17 & 4,4 & \\
\hline \multicolumn{8}{|l|}{ Disponibilidade diária de água } \\
\hline Sim & 490 & 78,4 & 190 & 83,2 & 292 & 75,5 & \multirow{2}{*}{, 022} \\
\hline Não & 135 & 21,6 & 40 & 16,8 & 95 & 24,5 & \\
\hline \multicolumn{8}{|l|}{ Ocupação do responsável } \\
\hline Tem trabalho & 261 & 41,6 & 99 & 41,6 & 162 & 41,6 & \multirow{2}{*}{,990 } \\
\hline Não tem trabalho ${ }^{2}$ & 366 & 58,4 & 139 & 58,4 & 227 & 58,4 & \\
\hline \multicolumn{8}{|l|}{ Escolaridade do responsável ${ }^{3}$} \\
\hline Baixa escolaridade & 438 & 70,1 & 153 & 64,6 & 285 & 73,5 & \multirow{2}{*}{,018 } \\
\hline Alta escolaridade & 187 & 29,9 & 84 & 35,4 & 103 & 26,5 & \\
\hline
\end{tabular}

${ }^{1} 10$ casos Não sabem/ Não respondeu; ${ }^{2}$ Incluindo aposentados e pensionistas; ${ }^{3} 02$ casos Não sabem/ Não respondeu 
Com relação às características demográficas e antropométricas dos escolares estudados, ao comparar os dois grupos de famílias analisados verificou-se uma homogeneidade na amostra, tanto para os dados de sexo e idade, quanto para peso e altura (Tabela 2). A faixa de idade de 0 a 4 anos correspondeu a $34,8 \%$ da amostra, enquanto que $65,2 \%$ das crianças tinham idade entre 5 e 9 anos.

Tabela 2. Características gerais da amostra segundo situação de vulnerabilidade social, Cuité-PB, 2012.

\begin{tabular}{|c|c|c|c|c|c|c|}
\hline \multirow[t]{2}{*}{ Variáveis } & \multicolumn{2}{|c|}{ TOTAL } & \multicolumn{2}{|c|}{$\begin{array}{l}\text { Famílias acima da } \\
\text { linha da pobreza }\end{array}$} & \multicolumn{2}{|c|}{$\begin{array}{l}\text { Famílias abaixo da } \\
\text { linha da pobreza }\end{array}$} \\
\hline & $\mathrm{N}$ & $\%$ & $\mathrm{~N}$ & $\%$ & $\mathrm{~N}$ & $\%$ \\
\hline \multicolumn{7}{|l|}{ Sexo } \\
\hline Masculino & 335 & 53,4 & 131 & 55 & 204 & 52,4 \\
\hline Feminino & 292 & 46,6 & 107 & 45 & 185 & 47,4 \\
\hline \multicolumn{7}{|l|}{ Idade } \\
\hline Média da idade & & & \multicolumn{2}{|c|}{$5,43( \pm 2,16)$} & \multicolumn{2}{|c|}{$5,42( \pm 2,25)$} \\
\hline \multicolumn{7}{|l|}{ Peso } \\
\hline Média de peso & & & \multicolumn{2}{|c|}{$21,76( \pm 7,19)$} & \multicolumn{2}{|c|}{$21,08( \pm 6,53)$} \\
\hline \multicolumn{7}{|l|}{ Altura } \\
\hline Média da altura & & & \multicolumn{2}{|c|}{$0,99( \pm 0,46)$} & \multicolumn{2}{|c|}{$0,97( \pm 0,47)$} \\
\hline
\end{tabular}

Ao analisar o estado nutricional das crianças, observou-se uma prevalência mais expressiva da eutrofia em ambos os grupos de famílias (Tabela 3). Ao comparar os resultados do indicador peso/idade segundo condição social, constatou-se que cerca de $20 \%$ das crianças estudadas foram classificadas com excesso de peso em ambos os grupos de famílias, enquanto que para o baixo peso verificou-se uma prevalência superior entre famílias classificadas abaixo da linha da pobreza.

Para o indicador altura/idade, que consegue avaliar perdas nutricionais em longo prazo, verificou-se um resultado diferente, pois os percentuais de risco e de baixa estatura eram semelhantes para crianças que residiam em famílias acima e abaixo da linha da pobreza.

Outro indicador relacionado com o estado nutricional adotado neste estudo é a análise de sinais clínicos de carências nutricionais. Na Tabela 3 é possível verificar a presença de 3 a 5 sinais de alterações clínicas relacionadas às deficiências nutricionais em crianças que convivem com famílias acima e abaixo da pobreza, sendo 20,4\% e 22,4\%, respectivamente. 
Tabela 3. Estado nutricional das crianças, segundo situação de vulnerabilidade social, CuitéPB, 2012.

\begin{tabular}{|c|c|c|c|c|c|c|c|}
\hline \multirow[t]{2}{*}{ Variáveis } & \multicolumn{2}{|c|}{ TOTAL } & \multicolumn{2}{|c|}{$\begin{array}{c}\text { Famílias acima } \\
\text { da linha da } \\
\text { pobreza }\end{array}$} & \multicolumn{2}{|c|}{$\begin{array}{c}\text { Famílias abaixo } \\
\text { da linha da } \\
\text { pobreza }\end{array}$} & \multirow[t]{2}{*}{$\begin{array}{l}\text { Valor } \\
\text { de } P\end{array}$} \\
\hline & $\mathrm{N}$ & $\%$ & $\mathrm{~N}$ & $\%$ & $\mathrm{~N}$ & $\%$ & \\
\hline \multicolumn{8}{|l|}{ Indicador Peso/ Idade } \\
\hline Baixo peso & 71 & 12 & 22 & 9,6 & 49 & 13,5 & \multirow{3}{*}{,348 } \\
\hline Eutrofia & 402 & 68,1 & 158 & 69,3 & 244 & 67,4 & \\
\hline Excesso de peso & 117 & 19,8 & 48 & 21,1 & 69 & 19,1 & \\
\hline \multicolumn{8}{|c|}{ Crianças com idade superior a 4 anos: Indicador Altura/Idade } \\
\hline Baixa estatura & 71 & 12 & 29 & 12,7 & 42 & 11,6 & \multirow{3}{*}{, 841} \\
\hline Eutrofia & 456 & 77,2 & 173 & 75,9 & 283 & 78,0 & \\
\hline Estatura elevada & 64 & 10,8 & 26 & 11,4 & 38 & 10,5 & \\
\hline \multicolumn{8}{|c|}{ Sinais clínicos de carências nutricionais } \\
\hline 0 a 2 sinais clínicos & 452 & 78,3 & 179 & 79,6 & 273 & 77,6 & \multirow{2}{*}{, 570} \\
\hline 3 a 5 sinais clínicos & 125 & 21,7 & 46 & 20,4 & 79 & 22,4 & \\
\hline
\end{tabular}

No entanto, ao comparar os grupos de risco de crianças com baixo peso para idade e excesso de peso, como mostra a Tabela 4, constatou-se que as crianças que apresentaram de 3 a 5 sinais carenciais e estavam inclusas em famílias abaixo da linha da pobreza apresentaram maior percentual de déficit de peso $(19,5 \%)$ do que excesso de peso (15,6\%). O contrário foi observado para as crianças pertencentes a famílias acima da linha da pobreza com as mesmas alterações clínicas, pois a prevalência de excesso de peso (20\%) se revelou expressivamente maior que a de baixo peso para a idade $(8,9 \%)$, mostrando que a presença de sinais de carências nutricionais também foi presente em crianças com características de peso opostas. 
Tabela 4. Estado nutricional segundo situação da vulnerabilidade social da família e presença de sinais clínicos de carências nutricionais, Cuité-PB, 2012.

Estado nutricional

Sinais clínicos de carências

nutricionais

\begin{tabular}{cccccc}
\hline Baixo peso & Eutrofia & $\begin{array}{c}\text { Excesso de } \\
\text { peso }\end{array}$ & $\begin{array}{c}\text { Valor } \\
\text { de } P\end{array}$ \\
\cline { 1 - 2 } $\mathrm{N}$ & $\%$ & $\mathrm{~N}$ & $\%$ & $\mathrm{~N}$ & $\%$
\end{tabular}

Famílias acima da linha da pobreza

0 a 2 sinais clínicos

3 a 5 sinais clínicos
18

10,2

4

8,9

122

69,

32

71,1

36

20,5

$9 \quad 20,0$

Famílias abaixo da linha da pobreza

0 a 2 sinais clínicos

3 a 5 sinais clínicos
$34 \quad 12,8 \quad 178 \quad 66,9 \quad 54 \quad 20,3$

$\begin{array}{llllll}15 & 19,5 & 50 & 64,9 & 12 & 15,6\end{array}$

\section{Discussão}

Os dados apresentados revelam que a população de escolares estudada encontra-se, em sua maioria, em situação nutricional adequada, segundo os indicadores utilizados. Porém, a presença de sobrepeso e obesidade, baixo peso ou risco de baixo peso e até os sinais de carências nutricionais atingem uma parcela importante das crianças, estejam elas inseridas em famílias abaixo ou acima da linha da pobreza.

O perfil observado é uma característica comum no atual processo de transição nutricional vivenciado no Brasil, onde o excesso de peso, a deficiência específica de nutrientes, a desnutrição e outras doenças crônicas coexistem na mesma comunidade. Segundo Batista Filho et al., ${ }^{2}$ a projeção dos resultados de estudos efetuados nas últimas três décadas é indicativa de um comportamento claramente epidêmico da obesidade. Assim, estabelece-se um antagonismo de tendências temporais entre desnutrição e obesidade, definindo uma das características marcantes do processo de transição nutricional do país.

Apesar da presença de ambos os perfis nutricionais nas crianças, baixo peso e sobrepeso, o segundo prevaleceu nos grupos de renda estudados. Para Campos e colaboradores, ${ }^{14}$ nota-se que o aumento do sobrepeso tem atingido todas as faixas etárias e níveis socioeconômicos. NettoOliveira et al., ${ }^{15}$ ao avaliar o sobrepeso e a obesidade nas crianças de 6 a 7 anos e 9 meses de idade, 
regularmente matriculadas em 24 escolas de Maringá, de diferentes classes econômicas, segundo o Critério de Classificação Econômica Brasil, da Associação Brasileira de Empresas de Pesquisa (ABEP), ${ }^{16}$ verificou que, entre crianças de nível econômico superior, a prevalência de excesso de peso foi nitidamente maior, sendo de 28,5\%, 20,5\% e 16,7\% para os níveis econômicos alto, baixo e médio, respectivamente.

A condição socioeconômica das famílias estudadas caracteriza o quadro de vulnerabilidade no município, colaborando para um desequilíbrio nas condições de saúde e bem-estar da população, especialmente no tocante ao acesso a alimentos diversificados e de qualidade. As condições ambientais são responsáveis por uma parcela significativa dentre os fatores determinantes da saúde infantil, com destaque para as deficiências nutricionais, a exemplo de situações desfavoráveis referentes ao acesso à água potável, destinação de dejetos (saneamento) e habitação, que contribuem para a morbidade e mortalidade significativa de crianças em países com estrutura sanitária insuficiente e inadequada, como o Brasil. ${ }^{17}$

Entre os indicadores socioeconômicos, a evolução do poder aquisitivo das famílias e a progressão da escolaridade dos seus membros repercutem diretamente na tendência secular das condições de saúde na infância. Do poder aquisitivo dependem, por exemplo, a disponibilidade de alimentos, a qualidade do ambiente e o acesso a serviços essenciais, como os de saneamento e os de assistência à saúde. ${ }^{18}$ Em um estudo realizado por Marinho, ${ }^{19}$ a partir da base de dados do SISVAN nos municípios da 1aㅡ Coordenadoria Regional de Saúde do Estado do Rio Grande do Sul em 2006, é possível perceber que o estado nutricional de uma população é resultante de vários fatores sociais, dentre eles, a educação. Uma vez deficiente, a escolaridade dos pais pode interferir negativamente no estado nutricional da população infantil. Conforme mostra Monteiro et al., ${ }^{20}$ ao analisar inquéritos antropométricos realizados em nível nacional, a frequência de crianças desnutridas tendeu a aumentar tanto com a diminuição do poder aquisitivo e da escolaridade materna, quanto com o menor acesso à assistência à saúde e ao saneamento.

Nesse contexto, importantes políticas sociais e de saúde vêm sendo implementadas nas últimas décadas com o objetivo de reduzir a pobreza, a insegurança alimentar e melhorar o acesso e a qualidade dos serviços de saúde, como o Programa Nacional de Alimentação Escolar - PNAE, o Programa Saúde na Escola - PSE, o Sistema Nacional de Vigilância Alimentar e Nutricional - SISVAN, a Estratégia Fome Zero e o então Programa Bolsa Família. ${ }^{21,22,23,24}$ Essas políticas e programas têm alcançado amplas coberturas populacionais num período de tempo relativamente breve, trazendo impactos importantes para a saúde e a nutrição infantil. ${ }^{25}$ Um estudo inédito realizado em 2.853 municípios revela que o programa de transferência de renda Bolsa Família teve contribuição decisiva para a queda da mortalidade de crianças menores de 5 anos, entre 2004 e 2009. A redução da mortalidade infantil nas cidades averiguadas chegou a 17\%. O estudo ainda apontou que a ação direta do Programa Bolsa Família na queda da mortalidade de crianças foi ainda maior quando a causa está relacionada à privação alimentar, ou seja, o programa foi 
responsável pela diminuição de $65 \%$ das mortes causadas por desnutrição e por $53 \%$ dos óbitos causados por diarreia. ${ }^{26}$

Os valores encontrados no presente estudo revelam a maior prevalência do excesso de peso sobre o baixo peso, independente do grupo de renda. Na população infantil, a prevalência de excesso de peso, nos últimos 30 anos, aumentou em todas as regiões brasileiras e em todos os extratos de renda, como também revelado na POF 2008/2009. ${ }^{1}$ O aumento da epidemia de obesidade tem sido observado em diversos países. Embora a prevalência de obesidade e de excesso de peso seja mais alta em países ricos como os EUA, observa-se que há um rápido aumento em vários países menos desenvolvidos entre adultos e crianças. Em crianças pré-escolares (0-5 anos de idade) nos países menos desenvolvidos, a prevalência de sobrepeso e obesidade tem crescido, inclusive no Brasil. ${ }^{27}$ A obesidade acomete populações, independentemente do estágio do ciclo da vida ou da condição socioeconômica. Importa destacar que quanto mais intenso e precoce é o seu surgimento, maior o risco de persistência e mais graves as comorbidades associadas, a exemplo das doenças cardiovasculares, hipertensão arterial, diabetes e alguns tipos de neoplasias. ${ }^{28}$

No que remete ao estado nutricional segundo o indicador altura/idade, utilizado apenas para crianças com idade acima de quatro anos, verificou-se elevado índice de crianças em risco para baixa estatura nos diferentes grupos, quando comparado às prevalências encontradas em nível nacional e regional. Segundo a POF 2008/2009, a baixa estatura, avaliada a partir do mesmo indicador entre crianças de 5 e 9 anos de idade, foi igual a $6,8 \%$ no Brasil e 7,9\% na região Nordeste. ${ }^{1}$ A Pesquisa Nacional Desenvolvimento Social - PNDS 2006 traz que o retardo de crescimento na infância concentra-se nos estratos sociais menos favorecidos, nesse caso, entre filhos de mães com um a três anos de escolaridade (13,6\%) ou sem escolaridade $(16,6 \%) \cdot{ }^{29}$ Orlonski et al., ${ }^{30}$ ao analisar 335 crianças entre 4 e 10 anos de idade, segundo indicador altura/idade, matriculadas no ensino básico do Centro de Atenção à Criança e ao Adolescente (CAIC) do município de Ponta Grossa, Paraná, constatou a presença de 6,9\% dessas em situação de baixa estatura.

Nesse sentido, destaca-se o forte aspecto do processo de transição nutricional no município de Cuité, onde, apesar do sobrepeso prevalecer em relação ao baixo peso segundo indicador peso/idade, as crianças estudadas ainda apresentam déficit de crescimento. O índice altura/idade expressa o crescimento linear de crianças e corresponde ao dado que melhor representa o efeito cumulativo de situações adversas sobre esta fase, ${ }^{13}$ para a qual, o consumo adequado de macro e micronutrientes tem um papel muito importante na promoção do crescimento físico. ${ }^{31}$

Assim, para Singh, ${ }^{32}$ as deficiências de vitamina A, ferro e zinco, por exemplo, ainda apresentam altas prevalências na maioria dos países em desenvolvimento, ocasionando diversos agravos à saúde dos indivíduos, uma vez que esses nutrientes são essenciais para o adequado funcionamento do organismo e, especialmente em crianças, para a otimização do processo de crescimento e desenvolvimento. 
Na sociedade contemporânea, a criança constitui um dos grupos da população mais vulneráveis à deficiência de micronutrientes, uma vez que são expostas a situações de alto risco para problemas nutricionais durante seu crescimento, desde a ingestão alimentar inadequada até a convivência com diferentes doenças infecciosas. ${ }^{33}$ Para Pedraza et al., ${ }^{34}$ a deficiência de micronutrientes está relacionada com uma série de efeitos deletérios na infância, com consequente aumento das taxas de morbimortalidade, entre outros agravos à saúde, reforçando ainda mais, como mostra o presente estudo, o risco nutricional dessa população fisiologicamente vulnerável. Por isso, essa etapa da vida representa um momento biológico que merece o máximo de atenção com relação à oferta de micronutrientes, em especial ferro, vitamina A e zinco, haja vista sua importância no baixo peso ao nascer e no déficit de estatura para o crescimento adequado.

Em um estudo de Pereira et al., ${ }^{35}$ que avaliou a presença de anemia em crianças na fase escolar a partir dos níveis séricos de hemoglobina, constatou-se que dos 267 escolares que compunham a amostra, mais da metade $(53,2 \%)$ era portadora de anemia, valor muito superior ao encontrado para os sinais clínicos de carências (21,6\%), que também sugerem a presença da anemia. Em outro estudo, De Paula et al. ${ }^{36}$ encontraram, também a partir dos níveis séricos de hemoglobina e retinol de crianças com até 5 anos de idade, aproximadamente $16 \%$ e $35 \%$ de anemia e de deficiência de vitamina $A$, respectivamente.

Levando em conta o alto custo e a dificuldade de análise de amostras de sangue para grandes estudos que avaliam os déficits de micronutrientes na população, destaca-se a semiologia como uma estratégia metodológica para triagem inicial. Em 2001, a Organização Pan-Americana de Saúde (OMS) salientou a palidez palmar como ferramenta de triagem inicial da anemia. ${ }^{37}$ No mesmo ano, a OMS reforçou a importância da utilização dos sinais clínicos em populações carentes com alta prevalência de anemia moderada e grave. ${ }^{38}$ Assim, considerando as significativas prevalências de crianças que apresentaram sinais de carências nutricionais, ressalta-se a relevância do diagnóstico clínico realizado neste estudo, evidenciando que, possivelmente, uma parcela maior de crianças pode apresentar deficiências nutricionais quando avaliadas a partir dos níveis séricos, uma vez que a presença de sinais clínicos identificados pode relacionar-se com quadros de deficiências mais avançadas.

A partir dos resultados apontados para a presença de sinais de carências nutricionais, observa-se a existência desses tanto em crianças com baixo peso como em com excesso. Assim, argumenta-se que a obesidade não ausenta os escolares de carências nutricionais específicas, mostrando que o aumento dos índices desta epidemia na população pode, na verdade, representar um problema complexo e de maior dimensão.

Ferreira et al. ${ }^{39}$ ao avaliar a dosagem de hemoglobina em um estudo com quilombolas no Estado de Alagoas, concluíram que o fato de a criança apresentar sobrepeso não a isenta de carências nutricionais específicas que comprometem seu crescimento, desenvolvimento e saúde. 
Leão et al., ${ }^{3}$ em uma revisão bibliográfica, também apresentaram estudos em que se constatou o consumo inadequado de zinco e cálcio em população obesa e hipertensa com resistência à insulina. A anemia, por exemplo, é a deficiência nutricional de maior magnitude no Brasil, acometendo, aproximadamente, $50 \%$ das crianças. Considerando essa problemática, o governo brasileiro tem realizado investimentos nos últimos anos com a fortificação de farinhas de trigo e milho com ferro e ácido fólico, a suplementação preventiva de ferro por meio do programa Saúde de Ferro e, mais recentemente, com a Estratégia NutriSUS, que instituiu, a partir de 2015, a fortificação da alimentação de crianças de creches públicas com um conjunto de micronutrientes. ${ }^{40}$

Nos últimos anos, estudos apontam as implicações epidemiológicas da deficiência de micronutrientes, comparando-as à de macronutrientes. A maioria dessas deficiências é subclínica, fenômeno chamado de "fome oculta". ${ }^{32,41}$ Estima-se que mais de dois bilhões de pessoas no mundo tenham alguma deficiência de vitaminas e minerais essenciais, principalmente de ferro, vitamina A, iodo e zinco, sendo que a maioria delas vive em países de baixa renda. ${ }^{42}$ A compreensão de como essas desigualdades conformam o quadro de saúde, particularmente na infância, é fundamental para formulação, implementação e avaliação de políticas públicas, uma vez que para situações desiguais, fazem-se necessárias intervenções diferenciadas. ${ }^{43}$

Assim, combinando os achados de estudos nacionais sobre as carências nutricionais com os de excesso de peso, pode-se afirmar que o Brasil apresenta uma dupla carga de doenças com origem na alimentação. ${ }^{44}$ Segundo Popkin et al., ${ }^{45}$ o déficit nutricional precoce, seguido de excessos, pode ser particularmente importante em países de baixa e média renda submetidos a mudanças sociais e econômicas rápidas. Esta evidência é altamente relevante e constitui uma forte justificativa para a prevenção da obesidade em populações que sofreram mudanças dramáticas no ambiente nutricional como uma consequência da transição nutricional.

\section{Considerações finais}

A presente pesquisa viabilizou a obtenção de dados que podem contribuir para a ampliação de informações que caracterizam o estado nutricional infantil. Por meio das informações fornecidas pelos responsáveis, verificou-se que, por se tratar de um município de pequeno porte, a maioria da população caracteriza-se como de risco de vulnerabilidade social, o que explica uma expressiva parcela dos escolares se encontrarem em risco nutricional, seja para baixo peso ou excesso de peso, característico da atual situação vivenciada no Brasil.

Assim, diante da transição nutricional observada, a deficiência específica de nutrientes e o aumento do sobrepeso e obesidade poderão ser colocados cada vez mais como problemas importantes que carecem de intervenções consistentes, inovadoras e condizentes com o complexo 
perfil epidemiológico, capazes de reduzir ainda mais os níveis de morbimortalidade infantil e, consequentemente, melhorar a qualidade de vida dos indivíduos.

A efetivação de pesquisas como esta apresenta grande relevância para a elaboração de políticas públicas eficazes, pois subsidiam com o conhecimento sobre a magnitude de problemas nutricionais. O município de Cuité precisa traçar estratégias de como alcançar os grupos sociais mais distintos possíveis com as políticas públicas locais, uma vez que, como mostrou este estudo, os grupos mais vulneráveis já não são os únicos atingidos com os problemas de saúde e nutrição.

No tocante à utilização da semiologia nutricional como método de avaliação das crianças, destaque-se sua importância como ferramenta para triagem inicial da deficiência específica de nutrientes, uma vez que, em condições desfavoráveis ou mesmo em estudos de grande porte, esta técnica torna-se um potencial sinalizador de déficits mais graves. Porém, a dosagem sérica de micronutrientes continua sendo o método mais eficaz na identificação de deficiências nutricionais, sendo esta uma limitação do estudo.

\section{Referências}

1. Instituto Brasileiro de Geografia e Estatística. Pesquisa de Orçamentos Familiares - POF - 20082009. Antropometria e estado nutricional de crianças, adolescentes e adultos no Brasil. Rio de Janeiro: IBGE; 2010. Disponível em: http://biblioteca.ibge.gov.br/visualizacao/livros/liv45419.pdf

2. Batista Filho M, Rissin A. A transição nutricional no Brasil: tendências regionais e temporais. Cad. Saúde Pública 2013; 19(Supl 1):181-91.

3. Leão ALM, Santos LCD. Consumo de micronutrientes e excesso de peso: existe relação?. Rev Bras Epidemiol. 2012; 15(1):85-95.

4. Batista Filho M, Souza AI, Miglioli TC, Santos MC. Anemia e obesidade: um paradoxo da transição nutricional brasileira. Cad. Saúde Pública 2008; 24(2):247-57.

5. Fidelis CMF, Osório MM. Consumo alimentar de macro e micronutrientes de crianças menores de 5 anos no Estado de Pernambuco, Brasil. Rev Bras Saúde Matern Infant. 2007; 7(1):63-74.

6. Pedraza DF, Rocha ACD, Sousa CPDC. Crescimento e deficiências de micronutrientes: perfil das crianças assistidas no núcleo de creches do governo da Paraíba, Brasil. Ciênc. Saúde Coletiva 2013; 18(1):3379-90.

7. Brasil. Comissão Nacional sobre Determinantes Sociais da Saúde. As causas sociais das iniquidades em saúde no Brasil. Rio de Janeiro: Editora Fiocruz; 2008. Disponível em: http://cmdss2011.org/ site/wp-content/uploads/2011/07/relatorio_cndss.pdf

8. Gubert MB, Santos LMP, Moura EC. Estratégias de diagnóstico nutricional rápido em populações. In: Silva GL, Taddei JA, Toloni MHA, Lang RMF. Nutrição em Saúde Pública. Rio de Janeiro: Rubio; 2011. 
9. Brasil. Ministério da Saúde. Vigilância alimentar e nutricional-SISVAN: orientações básicas para a coleta, processamento, análise de dados e informação em serviços de saúde. Brasília: Ministério da Saúde; 2004. 120p.

10. Duarte ACG. Avaliação nutricional: aspectos clínicos e laboratoriais. São Paulo: Atheneu; 2007. 607 p.

11. World Health Organization. Young people's health: a Challenge for Society. Report of a WHO study group on young people and health for all. Geneva: WHO; 1986. Technical Report Series, 731.

12. Brasil. Decreto n ${ }^{\circ}$ 6.917, de 30 de julho de 2009. Altera os arts. 18, 19 e 28 do Decreto no 5.209, de 17 de setembro de 2004, que regulamenta a Lei no 10.836, de 9 de janeiro de 2004, que cria o Programa Bolsa Família. Diário Oficial da União 31 jul. 2009. Disponível em: http://www.planalto.gov.br/ ccivil_03/_Ato2007-2010/2009/Decreto/D6917.htm

13. World Health Organization. WHO child growth standards: methods and development: length/ height-for-age, weight-for-age, weight-for-length, weight-for-height and body mass index-for-age. Geneva: WHO; 2006.

14. Campos LDA, Almeida PCD. Nível socioeconômico e sua influência sobre a prevalência de sobrepeso e obesidade em escolares adolescentes do município de Fortaleza. Rev Nutr. 2006; 19(5):531-538.

15. Netto-Oliveira ER, Oliveira AAB, Nakashima ATA, Rosaneli CF, Oliveira Filho A, Rechenchosky $\mathrm{L}$, et al. Sobrepeso e obesidade em crianças de diferentes níveis econômicos. Rev Bras Cineantropom Desemp Hum. 2010; 12(2):83-9.

16. Associação Brasileira de Empresa e Pesquisa. CCEB. Critério de Classificação Econômica Brasil. ABEP; 2008. Acesso em 13 mar 2016. Disponível em: http://www.abep.org/criterio-brasil

17. Mello-da-Silva CA, Fruchtengarten L. Riscos químicos ambientais à saúde da criança. Jornal de Pediatria 2005; 81(5 Supl.):205-211.

18. Martins MDFD, Costa JSD, Saforcada ET, Cunha MDC. Qualidade do ambiente e fatores associados: um estudo em crianças de Pelotas, Rio Grande do Sul, Brasil. Cad. Saúde Pública 2004; 20(3):710-718.

19. Marinho CL. Estado nutricional de crianças de 0 a 10 anos acompanhadas pelo Sistema de Vigilância Alimentar e Nutricional (SISVAN) na $1^{\circ}$ Coordenadoria Regional de Saúde do Estado do Rio Grande do Sul, Brasil [trabalho de conclusão de curso]. Florianópolis: Universidade Federal do Rio Grande do Sul. Faculdade de Medicina. Curso de Nutrição; 2008.

20. Monteiro CA, Benicio MHD’A, Konno SC, Silva ACF, Lima ALL, Conde WL. Causas do declínio da desnutrição infantil no Brasil, 1996-2007. Rev. Saúde Pública 2009; 43(1):35-43.

21. Brasil. Resolução/CD/FNDE no 38, de 16 de julho de 2009. Dispõe sobre o atendimento da alimentação escolar aos alunos da educação básica no Programa Nacional de Alimentação Escolar - PNAE. Disponível em: http://educacaointegral.mec.gov.br/images/pdf/res_cd_38_16072009.pdf

22. Jaime PC, Silva ACF, Lima AMC, Bortolini GA. Ações de alimentação e nutrição na atenção básica: a experiência de organização no Governo Brasileiro. Rev. Nutr 2011; 24(6):809-824.

23. Paes-Sousa R. Plano Brasil Sem Miséria: incremento e mudança na política de proteção e promoção social no Brasil. Brasília: Centro Internacional de Políticas para o Crescimento Inclusivo; 2013. (IPC-IG Working Paper; 113). 
24. Silva JG, Del Grossi ME, França CG, organizadores. Fome zero: a experiência brasileira. Brasília: Ministério do Desenvolvimento Agrário; 2010.

25. Victora CG, Aquino EML, Leal MC, Monteiro CA, Barros FC, Szwarcwald CL. Saúde de mães e crianças no Brasil: progressos e desafios [Internet]. Saúde no Brasil, 2. Disponível em: http://dms.ufpel. edu.br/ares/bitstream/handle/123456789/279/15\%20Sa $\%$ C3\%BAde $\% 20 \mathrm{de} \% 20 \mathrm{~m} \% \mathrm{C} 3 \%$ A3es $\% 20$ e $\% 20 \operatorname{crian} \%$ C $3 \%$ A 7 as $\% 20$ no $\% 20$ Brasil $\% 20$ progressos $\% 20 \mathrm{e} \% 20$ desafios.pdf? sequence $=1$

26. Brasil. Ministério da Saúde. Bolsa Família acelera redução da mortalidade infantil. [Internet] Portal Brasil 03 nov. 2013. Disponível em: http://www.brasil.gov.br/saude/2013/11/bolsa-familiainfluenciou-na-reducao-da-mortalidade-infantil

27. Hook JV, Altman CE, Balistreri KS. Global patterns in overweight among children and mothers in less developed countries. Public Health Nutr. 2012; 16(4):573-581.

28. Leal VS, Lira PIC, Oliveira JS, Menezes RCE, Sequeira LAS, Arruda Neto MA, et al. Excesso de peso em crianças e adolescentes no Estado de Pernambuco, Brasil: prevalência e determinantes. Cad Saúde Pública 2012; 28(6):1175-82.

29. Brasil. Ministério da Saúde. Pesquisa Nacional de Demografia e Saúde da Criança e da Mulher-PNDS 2006: dimensões do processo reprodutivo e da saúde da criança. Brasília: Ministério da Saúde; 2009.

30. Orlonski S, Dellagrana RA, Rech CR, Araújo EDS. Estado nutricional e fatores associados ao déficit de Estatura em crianças atendidas por uma unidade de ensino Básico de tempo integral. Rev Bras Crescimento Desenvolvimento Hum. 2009; 19(1):54-62.

31. Vitolo MR. Nutrição-da gestação ao envelhecimento. 2 ed. Rio de Janeiro: Rubio; 2014.

32. Singh M. Role of micronutrients for physical growth and mental development. Indian J Pediatr. 2004; 71(1):59-62.

33. Macêdo EMC, Amorim MAF, Silva ACS, Castro CMMB. Efeitos da deficiência de cobre, zinco e magnésio sobre o sistema imune de crianças com desnutrição grave. Rev Paul Pediatr. 2010; 28(3):329-36.

34. Pedraza DF, Rocha ACD, Sales MC. Deficiência de micronutrientes e crescimento linear: revisão sistemática de estudos observacionais. Ciênc Saúde Coletiva 2013; 18(11):3333-3347.

35. Pereira RC, Ferreira LOC, Diniz AS, Batista Filho M, Figueirôa JN. Eficácia da suplementação de ferro associado ou não à vitamina A no controle da anemia em escolares. Cad. Saúde Pública 2007; 23(6):1415-1421.

36. Paula WKAS, Caminha MFC, Figuerôa JN, Batista Filho M. Anemia e deficiência de vitamina A em crianças menores de cinco anos assistidas pela Estratégia Saúde da Família no Estado de Pernambuco, Brasil. Ciênc Saúde Coletiva 2014; 19(4):1209-1222.

37. Validez de los signos clínicos para la evaluación y clasificación de anemia en los niños menores de 5 años. In: Benguigui Y, Bossio JC, Fernández HR. Investigaciones operativas sobre atención integrada a las enfermidades prevalentes de la infância - AIEPI. Washington: Organização Pan-Americana de Saúde; 2001. p. 282-290. Disponível em: http://publicaciones.ops.org.ar/publicaciones/salud_ familiar_comunitaria/RM/cdresiliencia/investigaciones $\% 20$ operativas/aiepi-caratula-contenido.pdf 
38. World Health Organization. Special Programme for Research \& Training in Tropical Diseases. The Prevention and management of severe anaemia in children in Malaria: endemic regions of Africa. Geneva: WHO; 2001.98 p.

39. Ferreira HS, Lamenha MLD, Xavier Júnior AFS, Cavalcante JC, Santos AM. Nutrição e saúde das crianças das comunidades remanescentes dos quilombos no Estado de Alagoas, Brasil. Rev. Panam Saúde Pública 2011; 30(1):51-58.

40. Brasil. Ministério da Saúde. Secretaria de Atenção à Saúde. Departamento de Atenção Básica. Programa Nacional de Suplementação de Ferro: manual de condutas gerais. Brasília: Ministério da Saúde; 2013. 24 p. Disponível em: http://189.28.128.100/dab/docs/portaldab/publicacoes/manual_ferro2013.pdf

41. Ferraz IS, Daneluzzi JC, Vannucchi H, Jordão Junior AA, Ricco RG, Del Ciampo LA, et al. Nível sérico de zinco e sua associação com deficiência de vitamina A em crianças pré-escolares. Jornal de Pediatria 2007; 83(6):512-517.

42. World Health Organization. Guideline: use of multiple micronutrient powders for home fortification of foods consumed by infants and children 6-23 months of age. Geneva: WHO; 2011. 25 p. Disponível em: http://apps.who.int/iris/bitstream/10665/44651/1/9789241502047_eng.pdf

43. Assis AMO, Barreto ML, Santos NS, Oliveira LPM, Santos SMC, Pinheiro SMC. Desigualdade, pobreza e condições de saúde e nutrição na infância no Nordeste brasileiro. Cad Saúde Pública 2007; 23(10):2337-2350.

44. Araújo MC, Bezerra IN, Barbosa FS, Junger WL, Yokoo EM, Pereira RA, et al. Consumo de macronutrientes e ingestão inadequada de micronutrientes em adultos. Rev Saúde Pública 2013; 47(1 Suppl.):177S-189S.

45. Popkin BM. Contemporary nutritional transition: determinants of diet and its impact on body composition. Proc Nutr Soc. 2011; 70(01):82-91.

Recebido: $14 / 12 / 2015$

Revisado: $11 / 3 / 2016$

Aceito: 28/5/2016 